\title{
Comparação e Classificação de risco entre ETFS e seus índices de referência
}

\author{
Comparison and Classification of risk between ETFS and their reference indices \\ Pedro Paulo Melo Arantes ${ }^{\dagger *}$, Guilherme Santos Souza ${ }^{\dagger}$, Antonio Sergio Torres Penedo ${ }^{\dagger}$, Vinícius Silva \\ Pereira $^{\dagger}$
}

Como citar esse artigo. Arantes, PPM;

Souza, GS; Penedo, AST; Pereira, VS. Comparação e Classificação de risco entre ETFS e seus índices de referência Revista Mosaico. 2018 Jan./Jun.; 09 (1):XX-XX.

\begin{abstract}
Resumo
Exchange Traded Funds (ETFs) são fundos negociados em bolsa de valores que foram a poucos anos incorporados no mercado de capitais brasileiro, fato ocorrido apenas em 2004. Dessa forma há poucos estudos sobre ETF, o que possibilita várias abordagens sobre sua temática e comparabilidade entre ETF, fundos e índices. Dessa forma este trabalho buscou comparar e classificar o risco das ETFs utilizando a medida de desvio-padrão e Índice Sharpe. Para isso utilizou-se de uma amostra intraday para as ETFs brasileiras no mês de outubro, avaliando numa perspectiva de curto prazo a variabilidade das ETFs. Os resultados indicam que há maior variabilidade do valor das ETFs se comparados com seus índices de referência, ainda o valor do Índice Sharpe apresenta valor negativo para a maior parte das ETFs estudadas, indicando uma valorização abaixo do índice de referência quanto ao prêmio pelo risco das ETFs.

Palavras-Chave: Exchange Traded Funds; Fundos; Risco; Mercado de capitais.
\end{abstract}

\begin{abstract}
Exchange Traded Funds (ETFs) are exchange-traded funds that were incorporated into the Brazilian capital market only a few years ago, a fact that occurred only in 2004. Thus, there are few studies on ETFs, which allows for several approaches on their thematic and comparability between ETFs, funds and indices. Thus, this work sought to compare and classify the risk of ETFs using the measure of standard deviation and Sharpe index. For this purpose, an intraday sample was used for Brazilian ETFs in October, evaluating the variability of ETFs in a short-term perspective. The results indicate that there is a greater variability of the value of the ETFs compared to their reference indices, while the value of the Sharpe Index shows a negative value for most of the ETFs studied, indicating a valuation below the reference index for the risk premium of ETFs.

Keywords: Exchange Traded Funds; Funds, Risk; Capital markets.
\end{abstract}

\section{Introdução}

Exchange Traded Funds (ETFs) são fundos negociados em bolsas de valores e que foram a poucos anos incorporados no mercado de capitais brasileiro, tendo tido sua criação em 1990 no Canadá e em 1993 na bolsa americana. No Brasil, sua primeira abertura se deu em 2004 com o PIBB (Papeis Índice Brasil Bovespa), lançado em conjunto pelo BNDES e Banco Itaú, com o segundo ETF sendo lançado 4 anos mais tarde pela Barclays, posteriormente adquirida pela gestora americana Blackrock (Borges, Eid Jr \& Yoshinaga, 2012; Deville, 2008).

No Brasil segundo informações da B3 (antiga BM\&F Bovespa) atualmente há uma oferta de 15 ETFs, sendo que há apenas quatro gestoras destes ETFs atuantes na bolsa, sendo o Banco Itaú, BlackRock (iShares), o Banco do Brasil e a Caixa Econômica Federal. Dados disponíveis dos fundos mostram um crescimento de $30 \%$ no valor montante do patrimônio ao ano nos ETFs para a última década, e um aumento considerável na quantidade de transações ocorridas nos mercados financeiros desenvolvidos, como a bolsa de Nova York que chegou a ter três ETFs dos cincos principais ativos mais negociados em outubro de 2016 e na qual a ETF mais negociada movimentou U\$ 331 bilhões (Souza, 2016).

Dessa forma, vários estudos sobre ETFs foram desenvolvidos nos últimos anos, no entanto, a literatura sobre esses fundos ainda é recente se considerar a rápida expansão deste tipo de ativo financeiro (Borges, Eid Jr \& Yoshinaga, 2012; Milani, 2015). Quanto ao seu volume mundial, segundo a BlackRock (2017), há mais de US\$ 3,1 trilhões em patrimônio, demonstrando seu grande poder expressivo.

Os ETFs são ativos compostos por agrupamentos de ações que visam à replicação de um índice do mercado financeiro, mas com a facilidade da negociação de ações individuais. Assim e também com a importância que os fundos de índices possuem, esse trabalho propõe

Afiliação dos autores: † Universidade Federal de Uberlândia - UFU; 
comparar a variação intraday das ETFs com seus fundos de referências e classifica-las de acordo com seu risco (desvio-padrão) e Índice Sharpe.

A comparação proposta está ponderada ao curto prazo, visando à variabilidade dia a dia do índice, tal característica espera mostrar a diferenciação de liquidez entre curto e longo prazo, no qual se sabe que a composição dos fundos está associada à capacidade de reestruturação da carteira à medida que novos investidores entram e saem. Dando assim uma perspectiva sobre liquidez para investidores que prezam por investimentos com retornos mais rápidos e líquidos, uma vez que leva-se tempo para estabilizar mudanças ocorridas na composição dos fundos e das ETFs.

A seguir serão discutidas várias assertivas sobre o tema e uma explicação mais detalha dos fundos e ETFs. Posteriormente a metodologia a ser trabalhada, seguida dos resultados e as considerações finais.

\section{Referencial Teórico}

Segundo a Comissão de Valores Mobiliários (CVM, 2017), um fundo de investimento é um patrimônio pertencente a diversas pessoas, sendo destinados a investimentos de certos ativos e normalmente geridos por profissionais. A característica do fundo depende do tipo de ativo que este negocia e as regras relacionadas a este pela CVM.

No Brasil, fundos de investimentos são regulamentados na receita federal possuindo um CNPJ. Dessa forma, eles são obrigados a possuir um estatuto social registrado, contendo direitos e deveres de cada cotista, bem como informações relacionadas à sua organização e funções administrativas exigidas pela CVM (Meneses \& Mariano, 2011).

Meneses e Mariano (2011) ilustram alguns tipos de fundos de investimento:

- Curto prazo: composto por títulos de renda fixa curto prazo seja inferior a 360 dias, possuindo um prazo médio de carteira menor.

- Referenciado: sua rentabilidade está atrelada a um indexador financeiro; composta de títulos de renda fixa $(95 \%)$

- Renda Fixa: composto por títulos de renda fixa pré ou pós-fixados $(80 \%)$ em sua carteira de investimentos.

- Multimercados: fundos com operações de derivativos financeiros que tem como objetivo a alavancagem do patrimônio como estratégia de investimento.

- Ações: origem de ações de empresas negociadas em Bolsa de Valores.

Quanto aos Exchange Traded Funds (ETFs) este são definidos pela CVM como fundos de investimentos abertos, admitidos a negociação em bolsa de valores com o objetivo principal de obter uma performance relacionada ao comportamento de um indicador de referência, estes também podem ser conhecidos como Fundos de Índices, devido a essa característica.

Segundo a B3, os principais motivos que se destacam para se investir em ETFs são: a) Diversificação, devido a forma como as ETFs são montadas, elas contêm diversos ativos lastreados a cada um de seus papéis individualmente, possibilitando assim um aumento na quantidade de setores de aplicação de forma simples e rápida; b) Baixo custo, uma vez que o investidor não precisa custear todos os custos operacionais para cada ação que o fundo de índice representa, investindo diretamente em todos estes ao se adquirir a ETF correspondente, ainda, elas possuem taxas de administração mais baixas que os fundos tradicionais; c) Praticidade, por serem ativos que retratam índices, ao se aplicar na ETF o investidor já terá uma diversificação satisfatória em sua carteira, tornando a aplicação mais prática; e d) Transparência, comparado a fundos tradicionais, o investidor tem acesso muito mais fácil à quais ativos ele está aplicando seus recursos, pois todas as informações são divulgadas, além de poder acompanhar minuto a minuto a variação do preço do mesma.

No Brasil há desafios quanto a investimentos, a maior parte da poupança financeira doméstica está aplicada em ativos de curto prazo e/ou indexados à taxa de juros de curtíssimo prazo. Tal fato implica numa cultura focada em imediatismo e de tal forma, os fundos acabam por ter pouco tempo para se reestruturar antes que o investidor de curto prazo sinta os efeitos. Muitos investidores preferem os investimentos de curto prazo devido ao grande risco que o investimento de logo prazo possui, existindo assim um viés de curto prazo em decisões sobre investimento (Freitas, 2011).

O mercado doméstico brasileiro de títulos privados ainda é pouco desenvolvido e sem liquidez, tal característica faz o investidor muitas vezes ser obrigado a carregar o título até o vencimento. Dessa forma opções que ofereçam uma alternativa a esse perfil podem ser vantajoso dentro desse viés de investimento ocorrido (Freitas, 2011)

Por serem negociados na bolsa e os investidores poderem acompanhar em tempo real sua valorização, as ETFs propiciam uma oportunidade ótimos para investidores de curto prazo. Assim saber o que ocorre dia a dia e seu comportamento ao longo do tempo pode auxiliar o investidor a tomar melhor suas decisões. Portanto, entender a variação intraday pode dar pistas do que é considerada habitual ou não e como essa relação é esperada com os índices de referência que são a todo o momento o foco das ETFs. 


\section{Estudos relacionados}

Na literatura, há estudos que defendem os ETFs em relação aos índices quanto ao retorno e risco e outros que afirmam que os ETFs dão menor retorno, mas apresentam facilidades que compensam tal fato. Soares e Milani (2015) fizeram uma análise dos fundos de sustentabilidade e os compararam com os índices tradicionais. Utilizando o índice Sharpe e o modelo do CAPM os autores encontraram que todos os fundos apresentam um devido-padrão inferior ao do mercado e ainda ao analisar o índice Sharpe encontraram uma superioridade dos fundos Livres, seguidos dos fundos de sustentabilidade e ETFs de sustentabilidade. No entanto, destacam o fato de haver poucos ETFs ligados a índices de sustentabilidade, sendo isso uma limitação.

Quando se trata de comparabilidade ao índice geral do Ibovespa, Matos, Iquiapaza e Ferreira (2014) encontraram que todos os ETFs tiveram perdas em relação ao índice, bem como com suas referências. Aplicaram a metodologia do Value-at-Risk a 5\% com simulação histórica, conjugada à Expected Shortfall e os resultados sugerem tal aspecto pela dificuldade do gestor do fundo em replicar corretamente o respectivo indexador, o que pode ainda ser mais impactante ao se considerar o curto prazo.

Maluf e Medeiros (2014) investigaram o nível de risco da ETF do Ibovespa e do próprio índice entre maio de 2009 até julho de 2013 utilizando Value at Risk e Expected Shortfalls, utilizando ainda a técnica de bootstrap. Como resultados, os autores apontam que o investimento indireto na carteira de mercado para o estudo feito oferece mais risco aos agentes que o investimento direto aos ativos da carteira, justificando que as facilidades oferecidas pelas ETFs podem ser o principal diferencial.

Milani (2015) em sua tese encontrou que o retorno das ETFs varia significativamente em relação ao mercado, tendo maior impacto quando um retorno negativo ocorre do que quando um positivo. Ainda os investidores preferem alocar recursos em ETFs já superavaliadas, uma vez que a experiência prévia passa segurança.

Assim, a variabilidade e incerteza ocorrida nas ETFs faz com que o investidor não ouse e mantenhase onde se sinta seguro, tal característica é agravada pelo pouco desenvolvimento financeiro que ocorre no Brasil e consequentemente abre poucas oportunidades para inovações de mercado. Assim, analisar o intraday e apresentar as informações pode ajudar a diminuir incertezas e apresentar um comportamento normal entre as diversas ETFs existentes, aumentando a possibilidade de investimentos e consequentemente apoiando o desenvolvimento do mercado de capitais brasileiro.

A seguir serão discutidos os métodos utilizados para a elaboração deste artigo, posteriormente haverá apresentação dos resultados e por fim as considerações finais.

\section{Metodologia}

A fim de alcançar o objetivo proposto, esse trabalho recolheu dados intraday de todas as ETFs negociadas na B3 (Salvo pela It Now Spxi devido a disponibilidade de dados no momento da coleta). A amostra contou com as ETFs e seus índices de referência (índice relacionado), assim como apresentado na Tabela 1. Tais informações foram adquiridas da ProfitChart

Tabela 1. ETFs com seus índices de referência

\begin{tabular}{|c|c|c|c|}
\hline Nome da ETF (Razão Social) & Fundo & Código & $\begin{array}{l}\text { Código do } \\
\text { Índice } \\
\text { Relacionado }\end{array}$ \\
\hline Caixa ETF Ibovespa Fundo de Îndice & Caixaetfxbov & XBOV11 & \multirow{3}{*}{ IBOV } \\
\hline ISHARES IBOVESPA Fundo de Indice & Ishares Bova $\mathrm{Ci}$ & BOVA11 & \\
\hline ITNOW IBOVESPA Fundo de Indice & It Now Ibov & BovV11 & \\
\hline ITNOW PIBB IBRX-50 - Fundo de Indice & Pibb Ind Brasil 50 & PIBB11 & IBXI \\
\hline ISHARES IBRX - Indice Brasil (Ibrx-100) & Ishares Ibrx Indice Brasil & BRAX11 & IBXX \\
\hline ISHARES Indice Carbono efic. (ico 2) Brasil & Ishares Ind Carb Ef & ECOO11 & $\mathrm{ICO} 2$ \\
\hline ITNOW IDIV Fundo de Indice & It Now Idiv & DIVO11 & IDIV \\
\hline ITNOW IFNC Fundo de Indice & It Now Ifnc Fundo de Indice & FIND11 & IFNC \\
\hline ITNOW IGCT Fundo de Indice & It Now Igct Fundo de Indice & GOVE11 & IGCT \\
\hline IT NOW IMAT Fundo de Indice & It Now Imat & MATB11 & IMAT \\
\hline ITNOW ISE Fundo de Indice & It Now Ise Fundo de Indice & ISUS11 & ISEE \\
\hline BB ETF S\&P Dividendos Brasil Fundo de Indice & BB Etf S\&P & BBSD11 & \multirow{2}{*}{ ISPFUT } \\
\hline ISHARES S\&P 500 Fundo Inv Cotas & Ishares S\&P 500 & IVVB11 & \\
\hline ISHARES BMFBOVESPA Small Cap Fundo de Indice & Ishares $\mathrm{Smal} \mathrm{Ci}$ & SMAL11 & SMLL \\
\hline
\end{tabular}


Real Time, que é uma plataforma para investidores que exibe resultados do mercado financeiro

A amostra foi definida como sendo todos os dias de atividade da Bolsa para o mês de outubro, tal escolha foi feita devido à disponibilidade de dados na plataforma utilizada e o foco no curso prazo, especificamente na variação intraday, dessa forma, foram coletados dados intraday minuto a minuto (note que tais dados eram limitados a plataforma de coleta utilizada, o que nem sempre apresentava dados minuto a minuto) para 21 dias, sendo que no caso dos fundos relacionados ao IBOV havia dados para 14 dias.

Quanto à metodologia de análise, essa se deu em duas etapas: a) Cálculo, classificação e apresentação do risco por desvio-padrão, tal medida é de utilização clássica na literatura e representa o quão volátil um ativo é no período de estudo, foi feito dia a dia utilizando a variação intraday disponível. Nessa etapa foi percebido que nem todos os objetos de estudo possuíam ampla variação intraday, o que impossibilitou o cálculo para certos dias, ainda é importante ressaltar que a variação estimada foi feita entre o valor de abertura e fechamento da amostra intraday obtida; b) Estimativa do índice Sharpe, que é uma medida de desempenho de um ativo, sendo desenvolvido pelo Nobel de Economia de 1990 Willian Sharpe, o período de estudo e apresentação deste será dia a dia, utilizando os dados intraday disponíveis.
Equação de cálculo do índice Sharpe:

$$
I S=\frac{\text { Retorno do Fundo }- \text { Retorno Livre de Risco }}{\text { Desvio Padrão do Retorno do Fundo }}
$$

Onde: IS = Índice Sharpe

O retorno do fundo foi calculado pela média do retorno nas variações intraday ocorridas dia a dia, o retorno livre de risco usado foi baseado no índice de referência. Sabe-se que o retorno livre de risco é normalmente baseado na taxa Selic, no entanto, como medida de comparação adotou-se o índice dos fundos, uma vez que ao se calcular o índice separadamente e dividir cada ETF com seu índice de referencia o valor de comparação seria o mesmo. Desta forma-se, adotase o retorno livre de risco como o índice de referência, indicando assim se o fundo está acima ou abaixo do índice quanto ao retorno.

Devido a essa utilização como referência para o índice livre de risco, foi esperado que o índice Sharpe apresentasse um valor referente ao índice relacionado, ou seja, um índice Sharpe positivo significa que o ETF teve um retorno acima do índice de referência enquanto que um valor negativo representa o contrário. Por fim, o desvio-padrão foi a variação ocorrida no dia estudado.

Para a exibição dos dados, índices ou ETFs que possuíam poucos dados de variação intraday ou então tiveram pouca atividade (menos de 5 variações de abertura e fechamento intraday) durante o dia foram retiradas, uma vez que só é possível o cálculo do desvio-

Tabela 2. Média dos valores encontrados na análise intraday

\begin{tabular}{llcccc}
\hline Tipo & Ativo & Desvio-padrão Médio & Retorno Médio (dia) & Índice Sharpe & $\begin{array}{c}\text { Observações } \\
\text { Intraday } \\
\text { (soma dias) }\end{array}$ \\
\hline ETF & BOVV11 & 0.002373658 & $-0.02877389 \%$ & -0.0103 & 41 \\
ETF & SMAL11 & 0.000693533 & $0.00059707 \%$ & 0.0316 & 4386 \\
ETF & DIVO11 & 0.000638287 & $0.00097320 \%$ & -0.0101 & 1047 \\
ETF & ISUS11 & 0.000574501 & $-0.00487837 \%$ & -0.0291 & 879 \\
ETF & PIBB11 & 0.000441139 & $-0.00018652 \%$ & -0.0147 & 1394 \\
ETF & BOVA11 & 0.000401091 & $-0.00160633 \%$ & -0.0291 & 6158 \\
ETF & IVVB11 & 0.000375968 & $-0.00266113 \%$ & -0.0661 & 3341 \\
ETF & FIND11 & 0.000310592 & $0.00254768 \%$ & 0.0882 & 215 \\
ETF & BBSD11 & 0.000039331 & $-0.00098781 \%$ & -0.2266 & 157 \\
ETF & XBOV11 & & & & \\
ETF & BRAX11 & & & & \\
ETF & ECOO11 & Não houve variação intraday ou valores suficientes para o cálculo & \\
ETF & GOVE11 & & & & \\
ETF & MATB11 & & & & \\
\hline
\end{tabular}


padrão caso haja variabilidade.

A seguir haverá a apresentação e discussão dos resultados encontrados.

\section{Análise de resultados}

A fim de facilitar a classificação das ETFs quanto ao seu desvio-padrão e Índice Sharpe, foi feito uma média geral para o mês estudado (Outubro), utilizando o resultado diário obtido pela análise intraday. Dessa forma, no primeiro momento será exibida a média do desvio-padrão encontrado na análise dia-a-dia, como visto na Tabela 2.

A Tabela 2 foi classificada de acordo com o desvio-padrão, em ordem decrescente, exibindo assim o ativo com maior variabilidade ou risco em primeiro lugar. A ETF BOVV11 apresentou maior variabilidade, tal valor pode ser esperado ao se considerar que é a ETF com maior aumento em seu volume de negociação do mês de outubro de 2017 se comparado ao mês de outubro de 2016 segundo dados da B3, que chegou a mais de 1.3 bilhões de reais em 2017 e no ano de 2016 havia tido apenas 12 milhões, sendo possível usar a mesma lógica para a SMAL11 que em outubro de 2016 havia um volume negociado de 3.3 milhões e em 2017
91 milhões. No entanto, o ativo mais negociado do mês, a ETF BOVA11 que chegou a mais de 4.5 bilhões em volume negociado não apresentou grande desvio-padrão médio.

Quanto ao Índice Sharpe, apenas FIND11 e SMAL11 obtiveram um valor positivo, significando que apenas estes tiveram uma premiação positiva quanto ao risco sofrido se comparado ao índice de referência. No entanto, as outras ETFs tiveram um valor negativo no índice, indicando que o prêmio pelo risco relacionado ao índice de referência não está sendo positivamente avaliado e o retorno está abaixo do esperado para tal nível de risco. Tal achado pode ser plausível ao se considerar o baixo nível de variabilidade encontrado, sendo assim o investidor aceita que o prêmio pelo risco seja inferior devido ao baixo risco atribuído as ETFs ou devido às facilidades ofertadas ao se investir em ETFs.

Comparado o desvio-padrão médio da Tabela 2 e da Tabela 3, percebe-se que a maior parte das ETFs possuem um desvio-padrão maior que dos índices de referência, o valor dessa diferença é apresentado na Tabela 4, na qual foi formulada a partir do Teste $\mathrm{T}$ de médias para sua validação. Tal característica corrobora com o encontrado por Maluf \& Medeiros (2014) e por Matos, Iquiapaza \& Ferreira (2014), ainda ao se

Tabela 3. Desvio-padrão médio e Retorno médio dos Índices de Referência

\begin{tabular}{lcc}
\hline Ativo & Desvio-padrão médio & Retorno Médio \\
\hline ISPFUT & 0.000065707 & $0.00004079 \%$ \\
IBOV & 0.000291666 & $-0.00011884 \%$ \\
IBXL & 0.000317225 & $0.00051891 \%$ \\
IBXX & 0.000290352 & $0.00046540 \%$ \\
ICO2 & 0.000335272 & $0.00056228 \%$ \\
IDIV & 0.000316484 & $0.00042848 \%$ \\
IFNC & 0.000370779 & $0.00053184 \%$ \\
IGCT & 0.000289721 & $0.00041165 \%$ \\
IMAT & 0.000483132 & $0.00061826 \%$ \\
ISEE & 0.000304276 & $0.00051958 \%$ \\
SMLL & 0.000223587 & $0.00025410 \%$ \\
\hline
\end{tabular}


Tabela 4. Diferença entre o Desvio-padrão e Retorno das ETFs com seus Índices de Referência

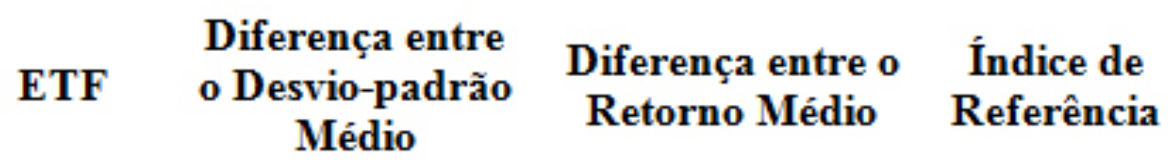

\begin{tabular}{cccc}
\hline BOVA11 & & -0.000014875 & IBOV \\
PIBB11 & & -0.000007054 & IBXL \\
DIVO11 & 0.000321803 & 0.000005447 & IDIV \\
FIND11 & -0.000060187 & 0.000020158 & IFNC \\
ISUS11 & 0.000270224 & -0.000053979 & ISEE \\
BBSD11 & -0.000026376 & -0.000010286 & ISPFUT \\
SMAL11 & & 0.000003430 & SMLL \\
\hline
\end{tabular}

Tabela 5. Análise Intraday para as ETFs

\begin{tabular}{|c|c|c|c|c|c|c|c|c|c|c|}
\hline \multirow[b]{2}{*}{ Dia } & \multicolumn{2}{|c|}{ Bovvil } & \multicolumn{2}{|c|}{ SMAL11 } & \multicolumn{2}{|c|}{ DIVOIl } & \multicolumn{2}{|c|}{ ISUS11 } & \multicolumn{2}{|c|}{ PIBB11 } \\
\hline & DP & IS & DP & IS & DP & IS & DP & IS & DP & IS \\
\hline $31 / 10 / 2017$ & 0.001083644 & -0.5530 & 0.000113378 & 0.1868 & 0.000185200 & -0.0472 & & & 0.000326484 & 0.0191 \\
\hline $30 / 10 / 2017$ & & & 0.001318777 & 0.0643 & & & 0.000354673 & -0.0393 & 0.000493515 & -0.0527 \\
\hline $27 / 10 / 2017$ & 0.002796068 & -0.5751 & 0.000520927 & 0.0053 & & & 0.000148969 & -0.1016 & 0.000229582 & 0.0403 \\
\hline $26 / 10 / 2017$ & & & 0.000209936 & 0.1932 & & & 0.000068658 & 0.3103 & 0.000276545 & 0.2220 \\
\hline $25 / 10 / 2017$ & 0.003766142 & 0.3207 & 0.000436520 & 0.0549 & 0.000041184 & -0.0213 & & & 0.000494623 & -0.1683 \\
\hline $24 / 10 / 2017$ & & & 0.000162948 & 0.0409 & 0.000112243 & -0.1493 & 0.000529162 & 0.0000 & 0.000259081 & 0.1083 \\
\hline $23 / 10 / 2017$ & 0.002813376 & -0.5758 & 0.000267935 & 0.0635 & 0.000132507 & 0.2371 & 0.003072935 & -0.1315 & 0.000411439 & -0.0864 \\
\hline $20 / 10 / 2017$ & 0.002278349 & -0.4988 & 0.000584632 & -0.0013 & 0.000081217 & -0.1816 & 0.001901906 & -0.1694 & 0.000327787 & -0.0261 \\
\hline $19 / 10 / 2017$ & 0.004200161 & 0.3812 & 0.000266301 & 0.0132 & 0.000396570 & 0.0798 & 0.002668587 & -0.1116 & 0.000196053 & 0.1981 \\
\hline $18 / 10 / 2017$ & 0.002961316 & 0.7054 & 0.000432840 & -0.0469 & 0.000623034 & -0.1790 & & & 0.000267017 & -0.2849 \\
\hline $17 / 10 / 2017$ & & & 0.000203803 & 0.0525 & 0.000701314 & 0.0703 & & & 0.000248042 & -0.2186 \\
\hline $16 / 10 / 2017$ & 0.000449766 & -0.5500 & 0.001794607 & -0.0077 & & & 0.000886157 & 0.1662 & 0.000420973 & 0.1983 \\
\hline $13 / 10 / 2017$ & & & 0.001657779 & -0.0386 & 0.000525901 & 0.4861 & 0.000279043 & 0.1933 & 0.000224063 & -0.2460 \\
\hline $11 / 10 / 2017$ & 0.001014104 & 0.6876 & 0.000166806 & -0.0304 & 0.000719805 & 0.1821 & & & 0.000423452 & 0.1904 \\
\hline $10 / 10 / 2017$ & & & 0.001568408 & -0.0342 & 0.003387454 & 0.0655 & 0.000863926 & -0.0533 & 0.000522268 & -0.2913 \\
\hline 09/10/2017 & & & 0.000335999 & 0.1633 & 0.000948596 & -0.1305 & & & 0.000563794 & 0.0214 \\
\hline $06 / 10 / 2017$ & & & 0.000347238 & -0.1433 & 0.000773314 & -0.2123 & 0.000865472 & -0.0031 & 0.000512906 & -0.0998 \\
\hline $05 / 10 / 2017$ & & & 0.000879747 & 0.1406 & 0.000546489 & 0.1086 & 0.000425025 & -0.4097 & 0.000434078 & 0.0025 \\
\hline $04 / 10 / 2017$ & & & 0.001306438 & -0.1201 & 0.000299176 & -0.2377 & & & 0.001077516 & -0.0572 \\
\hline 03/10/2017 & & & 0.001415510 & -0.0187 & 0.003732860 & -0.0410 & & & 0.001092053 & 0.0504 \\
\hline $02 / 10 / 2017$ & & & 0.000573655 & 0.1272 & 0.000197173 & -0.2011 & & & 0.000462638 & 0.1715 \\
\hline
\end{tabular}




\begin{tabular}{|c|c|c|c|c|c|c|c|c|}
\hline \multirow[b]{2}{*}{ Dia } & \multicolumn{2}{|c|}{ BOVAll } & \multicolumn{2}{|c|}{ IVVB11 } & \multicolumn{2}{|c|}{ FIND11 } & \multicolumn{2}{|c|}{ BBSDII } \\
\hline & DP & IS & DP & IS & DP & IS & DP & IS \\
\hline $31 / 10 / 2017$ & 0.000454877 & 0.0462 & 0.000674193 & -0.1349 & 0.000119565 & 0.6623 & & \\
\hline $30 / 10 / 2017$ & 0.000501574 & -0.0494 & 0.000112338 & 0.0612 & 0.000631951 & -0.2908 & 0.000070485 & -0.3709 \\
\hline $27 / 10 / 2017$ & 0.000481353 & -0.0751 & 0.000278769 & -0.3388 & 0.000970002 & -0.3269 & & \\
\hline $26 / 10 / 2017$ & 0.000471274 & -0.1342 & 0.000424857 & 0.0749 & 0.000063447 & 0.7433 & & \\
\hline $25 / 10 / 2017$ & 0.000485368 & -0.1645 & 0.000246146 & 0.0028 & & & & \\
\hline $24 / 10 / 2017$ & 0.000408979 & -0.0115 & 0.000248655 & 0.0815 & & & & \\
\hline $23 / 10 / 2017$ & 0.000431241 & -0.0743 & 0.000238204 & -0.1190 & & & & \\
\hline $20 / 10 / 2017$ & 0.000397007 & -0.0098 & 0.000217708 & 0.0081 & & & & \\
\hline $19 / 10 / 2017$ & 0.000335639 & -0.0552 & 0.000257428 & -0.0948 & 0.000147266 & 0.1673 & & \\
\hline $18 / 10 / 2017$ & 0.000301791 & 0.2709 & 0.000345123 & -0.0599 & & & 0.000084517 & -0.5513 \\
\hline $17 / 10 / 2017$ & 0.000327928 & -0.0248 & 0.000160831 & -0.3054 & & & & \\
\hline $16 / 10 / 2017$ & 0.000332509 & 0.0346 & 0.001125080 & -0.1084 & 0.000077975 & 0.3070 & 0.000090728 & -0.0650 \\
\hline $13 / 10 / 2017$ & 0.000301958 & -0.1285 & 0.001154015 & -0.0820 & & & & \\
\hline $11 / 10 / 2017$ & 0.000383782 & -0.0324 & 0.000216006 & 0.0575 & & & 0.000102103 & -0.3958 \\
\hline $10 / 10 / 2017$ & & & 0.000624835 & -0.0843 & 0.000272241 & -0.4828 & & \\
\hline $09 / 10 / 2017$ & & & 0.000171510 & -0.0944 & 0.001604611 & 0.2688 & 0.000057244 & 0.4269 \\
\hline $06 / 10 / 2017$ & & & 0.000349277 & -0.1084 & 0.000222328 & -0.6597 & 0.000227376 & -0.3058 \\
\hline $05 / 10 / 2017$ & & & 0.000309159 & -0.0303 & 0.000114707 & -0.2893 & 0.000135932 & -0.2691 \\
\hline $04 / 10 / 2017$ & & & 0.000204842 & -0.2962 & 0.000128088 & 0.4815 & & \\
\hline $03 / 10 / 2017$ & & & 0.000186475 & 0.0240 & 0.001781202 & 0.3023 & & \\
\hline $02 / 10 / 2017$ & & & 0.000349884 & 0.1589 & 0.000389040 & 0.2642 & 0.000057561 & -0.2819 \\
\hline
\end{tabular}

considerar o valor do Índice Sharpe pode se inferir que há uma perda ao valor do prêmio ao risco, sugerindo que apesar das facilidades encontradas ao se investir nas ETFs há um risco superior do que se investir na carteira do índice relacionado.

Analisando a Tabela 4, que apresenta a diferença das médias e considerando o Teste $\mathrm{T}$ feito a $5 \%$ de significância, apenas 4 das 9 ETFs tiveram um desvio padrão médio estatisticamente diferente, na qual FIND11 e BBSD11 apresentaram um desvio-padrão maior que seus índices de referência, confirmando que para estes 2 ETFs há um maior risco. Quanto a diferença de retorno, apenas 3 das 7 ETFs que tiveram diferença estatística, apresentaram retorno médio superior aos seus índices de referência para o período e amostra utilizada.

$\mathrm{Na}$ Tabela 5 é possível conferir a análise intraday para oNa Tabela 5 é possível conferir a análise intraday para o mês estudado. As observações em branco se referem a pouca ou nenhuma variabilidade ocorrida no dia, impedindo o cálculo.

\section{Considerações Finais}

Este trabalho buscou estimar o risco das ETFs via desvio-padrão e Índice Sharpe, classificando-as em ordem de risco. Foram utilizados dados intraday para fazer uma avaliação dia a dia para o mês de outubro de 2017. A amostra utilizada permitiu fazer a avaliação de 14 das 15 ETFs disponíveis atualmente no mercado financeiro brasileiro.

Utilizando dados intraday da ProfitChart Real Time, percebeu baixa variabilidade geral das ETFs, destacando apenas aquelas que lideram o volume de negociação ou de rápida expansão, como a BOVV11 e SMAL11. Ainda se mostrou que as ETFs no geral possuem maior desvio-padrão, salvo pela FIND11 e BBSD11, dessa forma pode sugerir que há maior 
risco ao se investir nas ETFs do que em uma carteira composta pelos ativos representados pelo seu índice de referência.

Quanto ao Índice Sharpe, este foi calculado baseado no índice de referência da ETF, este mostrou grande variabilidade ao longo dos dias, tendo uma média negativa para o mês em todas as ETFs, com exceção da SMAL11 e FIND11. Conclui-se, portanto, que o prêmio pelo risco adicional ao índice não é suficiente, e que possivelmente há outros fatores além do prêmio que levam o investidor a preferir o investimento, como possivelmente as facilidades oferecidas pelas ETFs.

Dessa forma, espera-se ter contribuído ao elencar pistas sobre o recente fenômeno das ETFs e sua preferência quanto ao risco, além de dar indícios sobre quais são as mais arriscadas.

Quanto às limitações, a disponibilidade e confiabilidade dos dados pode ser um fator crucial, principalmente ao se trabalhar com dados intraday, que são de difícil obtenção, sendo esta a principal limitação deste trabalho, uma vez que uma análise de mais meses e com melhor estimativa intraday seria o ideal, no entanto o volume de negociações também impede uma comparação mais robusta se comparado a mercados financeiros mais desenvolvidos.

Por fim, para estudos relacionados investigar os principais motivos relacionados ao 'Boom' das ETFs e elencar a importância real do risco frente aos outros motivos estudados na literatura. Ainda a reaplicação deste estudo de modo a superar as limitações aqui encontradas, calculando os custos reais de investimento em fundos e ETFs e comparar o ganho real do investidor.

\section{Referências}

B3 - ANTIGA BM\&FBOVESPA SOBRE SEGMENTOS DE LISTAGEM. Disponível em: <http://www.bmfbovespa.com.br/pt br/listagem/acoes/ segmentos-de-listagem/sobre-segmentos-de-listagem/>. Acesso em: $26 \mathrm{dez}$ 2017

BLACKROCK - THE CASE FOR ETFS IN THREE CHARTS. Disponível em: <https://www.blackrock.com/br/insights/the-case-for-etfs $>$. Acesso em: $26 \operatorname{dez} 2017$.

BORGES, E. C., EID JR, W., \& YOSHINAGA, C. E. Exchange traded funds versus fundos indexados no Brasil. Revista de Finanças Aplicadas, v. 1, n. 0 , p. 1-18, 2012.

COMISSÃO DE VALORES MOBILIÁRIOS (CVM) - FUNDOS. Disponível em: <http://www.cvm.gov.br/menu/regulados/fundos/sobre. html>. Acesso em: $26 \mathrm{dez} 2017$.

DEVILLE, L. Exchange traded funds: History, trading, and research. In: Handbook of Financial Engineering. Springer US, p. 67-98, 2008.

FREITAS, M. C. P. Financiamento de longo prazo no Brasil: avanços e desafios. Financiamento das Corporações, p. 153, 2011.

MALUF, Y. S.; MEDEIROS, O. R. Value-at-Risk of Brazilian ETFs with Extreme Value Theory Approach. Revista de Finanças Aplicadas, v. 1, n. 1, p. $1-34,2014$

MATOS, G. A. S., IQUIAPAZA, R. A., \& FERREIRA, B. P. Análise da exposição a perdas dos ETFs brasileiros conforme as técnicas de avaliação de risco de mercado Value at Risk (VaR) e Expected Shortfall (ES). BBRBrazilian Business Review, v. 11, n. 4, 2014.

MENESES, A; MARIANO, F. Mercado Financeiro: Teoria, questões comentadas e mais de 100 questões propostas. 2011.

MILANI, B. O desvio do preço dos exchange-traded funds brasileiros: uma análise baseada na correlação local. Tese de Doutorado. Universidade Federal de Santa Maria. 2015.

SOARES, E. D. M., \& MILANI, B. Fundos De Investimento Tradicionais e ETFs Ligados a Índices De Sustentabilidade, Responsabilidade Social E Governança Corporativa Apresentam Performance Superior? Revista de Gestão, Finanças e Contabilidade, 5(0), 26-42, 2015

SOUZA, R. P.. Análise de desempenho de estratégias com carteiras formadas por ETFs no Brasil nos anos de 2012 a 2016. 2016. Dissertação (Mestrado em Administração) - FECAP, São Paulo. 